\title{
Philosophiques
}

\section{Contribution à la disputatio de Jean-François Kervégan : Que faire de Carl Schmitt?}

\section{Catherine Colliot-Thélène}

Volume 39, numéro 2, automne 2012

URI : https://id.erudit.org/iderudit/1013700ar

DOI : https://doi.org/10.7202/1013700ar

Aller au sommaire du numéro

Éditeur(s)

Société de philosophie du Québec

ISSN

0316-2923 (imprimé)

1492-1391 (numérique)

Découvrir la revue

Citer ce document

Colliot-Thélène, C. (2012). Contribution à la disputatio de Jean-François Kervégan : Que faire de Carl Schmitt ?. Philosophiques, 39(2), 469-473.

https://doi.org/10.7202/1013700ar d'utilisation que vous pouvez consulter en ligne.

https://apropos.erudit.org/fr/usagers/politique-dutilisation/ 


\title{
Contribution à la disputatio de Jeen-François Kevégan: Que faire de Carl Schmitt?
}

\author{
CATHERINE COLLIOT-THÉLÈNE \\ Université de Rennes-1 \\ catherine.colliot@univ-rennesı.fr
}

L'ouvrage que Jean-François Kervégan a publié l'an passé sous le titre Que faire de Carl Schmitt? est une réussite rare, dans la mesure où peuvent en tirer profit aussi bien des lecteurs curieux, qui n'auraient jusqu'à présent rien lu de cet auteur, que des spécialistes, philosophes, politistes ou juristes, qui connaissent déjà tout ou partie de son œuvre et l'ont utilisée, commentée ou discutée dans leurs propres travaux. Cet exploit tient à trois qualités, qui caractérisent tous les travaux de Jean-François Kervégan: la pédagogie, la densité de la pensée et de l'expression, et une remarquable érudition. À quoi il faut encore ajouter une honnêteté scrupuleuse, laquelle lui permet d'adopter, par rapport aux controverses qu'ont suscitées, en France et ailleurs, les réceptions de l'œuvre de Schmitt, une attitude dont on se convainc, à le lire, qu'elle est la seule qui puisse justifier de s'intéresser à cette œuvre, à savoir : reconnaître un statut authentiquement théorique aux écrits de Carl Schmitt (du moins, à la plus grande partie de ses écrits), sans rien taire de son engagement politique au service du régime nazi, et sans craindre non plus de montrer les liens qui existent entre cet engagement et certaines de ses positions théoriques.

Le titre de l'ouvrage exprime bien la question centrale qui commande la présentation que Jean-François Kervégan fait de l'œuvre de Schmitt: «que faire de Carl Schmitt? ", c'est-à-dire d'abord, cela va de soi, peut-on encore en faire quelque chose, et quoi? Mais plus précisément: il ne s'agit pas seulement d'établir que l'œuvre de Schmitt mérite d'être traitée comme une œuvre de théorie (et non comme un simple document qui n'aurait d'intérêt que pour les historiens de l'idéologie nazie), mais aussi et surtout de dégager dans cette œuvre des concepts et des thématiques, s'ils existent, qui peuvent nous aider à penser notre actualité. Ce que Jean-François fait dans la seconde partie de son ouvrage, en sélectionnant cinq thèmes: la théologie, la normativité, la légitimité, le politique et le monde. Les remarques qui suivent portent avant tout sur les deux derniers thèmes.

Par delà les évolutions et les tournants apparents des intérêts de Schmitt, Jean-François Kervégan repère quelques thématiques constantes, parmi lesquelles sont tout particulièrement soulignées: I) la critique de l'argumentation morale ou humanitaire en politique, et 2) une interrogation sur le concept de politique, depuis l'époque où l'État en déterminait essentiellement le contenu jusqu'à la "péremption de la Staatlichkeit (étatité) " que Schmitt diagnostiquait en son temps. Cette critique et cette interrogation

\section{PHILOSOPHIQUES 39/2 - Automne2012, p. 469-473}


rencontrent une résonance certaine dans notre actualité, et avec elles les réflexions schmittiennes sur l'histoire du concept de la «guerre juste", sur les formes d'hégémonie qui utilisent à leur profit les organisations internationales, et sur la rhétorique humaniste qui habillent souvent ces usages et mésusages. Il est salutaire d'interpréter dans cette perspective la fameuse « définition" (ce n'était en vérité qu'un critère d'identification) schmittienne du politique par la distinction ami/ennemi, plutôt que de lui opposer doctement un concept de la politique-philia dont les capacités analytiques sont en raison inverse des mérites moraux. La première thématique (la critique de l'argumentation morale en politique) m'inspire la remarque qui suit. Le point d'articulation entre morale et politique réside dans le droit, et toute la question est ici de savoir si nous saisissons mieux ce qu'il en est de la réalité du droit en privilégiant son rapport à la morale ou bien à la politique. Kervégan souligne que l'un des principaux intérêts de la pensée schmittienne est de nous inciter à prendre en considération ce qu'il nomme le «moment politique du droit», un point que je lui accorde bien volontiers. Mais il dit aussi avoir acquis aujourd'hui la conviction qu'il est nécessaire de reconnaitre sur le plan juridique et judiciaire de l'organisation des sociétés une plus grande autonomie que celle que Schmitt (bien que juriste) lui accordait. Cette ouverture "au delà» de Schmitt m'amène à poser la question suivante: reconnaître une autonomie (relative, il va de soi) au droit implique$\mathrm{t}$-il de repenser les rapports entre droit, politique et morale ? Ou bien devonsnous considérer que les questions d'ordre moral, quelle que soit la manière dont on les définit, doivent rester hors champ dans les théories de la politique et du droit? Quel sort doit-on alors réserver aux «droits de l'homme ", qui, pour Schmitt, ne pouvaient apparaitre que comme une ingérence de considérations morales dans le droit et la politique, et qui, pourtant, aujourd'hui sinon à son époque, sont bien dans une certaine mesure institutionnalisés (à travers les "déclarations", leur inscription dans les constitutions, et l'existence de diverses instances judiciaires, nationales et supra-nationales)? Quelle est la place des droits de l'homme, ou, comme on préfère le dire aujourd'hui, des droits humains, dans le fonctionnement «normal» d'un ordre juridique établi?

Ma seconde question concerne la manière dont notre monde est monde. Schmitt contestait, on le sait, la possibilité d'une unification politique du monde. L'humanité, affirmait-il sans ambages, n'est pas un concept politique; un monde politiquement unifié serait un monde d'où la politique aurait disparu. "Le monde politique n'est pas un universum, mais, si l'on peut dire, un pluriversum.» Persuadé cependant que l'époque était révolue où cette pluralité était avant tout déterminée par la division entre les États territoriaux, Schmitt a cherché un nouveau pluralisme du côté des empires, c'est-à-dire du partage du monde entre espaces soumis à des puissances hégémoniques. Ce schéma pouvait prétendre à quelque pertinence au temps de la Guerre froide, mais l'effondrement de l'empire soviétique l'a rendu 
caduc. Qu'en est-il donc aujourd'hui ? Le monde est-il unifié, sur le plan juridique et politique, du fait que les conflits ne passent plus essentiellement entre les États ou entre les empires? Le monde contemporain est-il «devenu pour un temps, et pour la première fois depuis les Temps modernes, politiquement monopolaire », comme le suggère Kervégan (p. 206) en interprétant les phénomènes contemporains du terrorisme et de la lutte contre le terrorisme comme le symptôme de cette disparition tendancielle du politique, et des dangers qu'elle recèle. Ces dangers, est-il précisé, ne résident pas dans la disparition du conflit, mais dans l'illusion de sa négation, qui ouvre la voie à des formes $\mathrm{d}^{\mathrm{N}}$ " inimitié » dont la dynamique exterminatrice pourrait l'emporter sur tous les conflits de l'histoire passée. Contre les rêves d'une politique du consensus et de l'accord rationnel (on comprend que Habermas, notamment, est visé), Kervégan propose donc, à travers Schmitt, une réhabilitation du conflit. Ce qui l'amène à évoquer la possibilité d'un autre humanisme, un humanisme modeste qui s'accommode du conflit et renonce donc à l'idéal d'un monde définitivement pacifié, mais qui serait pourtant "un monde humain, et non un monde travaillé par la tentation d'éliminer une fois pour toutes les ennemis de l'humanité» (207).

Je souscris à cette volonté de légitimer le conflit, et au souhait sur lequel se termine l'ouvrage de Kervégan: "nous avons besoin de penseurs du dissentiment ». Mais aussi bien la manière dont Schmitt interprète l'êtremonde de notre monde que les brèves remarques de Kervégan sur la situation présente me laissent sur ma faim. La raison en est que le triptyque politique-morale-droit, ou (en admettant que l'on ait écarté la morale) le diptyque politique-droit, laisse de côté un facteur essentiel dans la constitution de l'unité du monde forgée par l'action des hommes, à savoir l'économie. Si l'on fait exception des remarques, au demeurant intéressantes, sur lesquelles se termine la Notion de politique, Schmitt a accordé peu d'attention aux dimensions économiques des logiques sociales, notamment au rôle qui leur revient dans la "péremption de la Staatlichkeit». La pensée économique n'était pour lui finalement qu'une variante de la pensée technique, c'est-à-dire d'une interprétation de l'histoire qui ignore "le problème crucial de l'ordre du monde ", lequel «est toujours un problème politique ». Dans le précis qu'il a écrit pour introduire cette disputatio, Kervégan évoque le fait que sa première lecture de Schmitt (il s'agissait du seul livre disponible en français à cette époque, La notion de politique, réunie dans un volume avec la Théorie du partisan) a été motivée par sa recherche d'une théorie du politique qu'il ne trouvait pas chez Marx. Nous appartenons à la même génération, notre jeunesse intellectuelle et politique a été assez comparable, nous étions l'un et l'autre des lecteurs assidus de Marx, et j'ai commencé à lire Schmitt pour les mêmes raisons. Il me paraît cependant que la théorie du politique que l'on peut trouver chez Schmitt souffre (entre autres choses) d'un défaut symétriquement opposé à celui que l'on peut reprocher à Marx. Si ce dernier a négligé les dimensions juridiques et politiques de la socialité 
pour avoir consacré l'essentiel de ses travaux théoriques à l'analyse des structures et dynamiques de l'économie capitaliste, Schmitt, à l'inverse, a focalisé son attention sur les rapports entre droit et politique, et négligé le rôle de l'économie dans les transformations des sociétés. Marx, précisément, ou une théorie économique critique qui s'en inspire de près ou de loin, permet d'appréhender ces transformations sous l'angle de l'économie, sans partager la représentation libérale, naïvement irénique ou sciemment mystificatrice, des relations économiques. Or une telle appréhension me paraît nécessaire pour rendre compte de la mondialisation contemporaine, dans ses dimensions juridique et politique aussi bien que dans sa dimension économique. Notre monde est bien Un, mais son unité est une unité dans la pluralité. En d'autres termes: il n'est pas monopolaire, pas même politiquement, parce que les relations de pouvoir qui donnent forme à ce monde ne se jouent plus seulement à un niveau que l'on puisse identifier comme politique en distinguant clairement celui-ci du juridique ou de l'économique. Elles se jouent en vérité à la fois dans tous ces domaines, entre lesquels les frontières sont de plus en plus indécises. Le concept moderne du politique perd en précision dès lors qu'il n'est plus arrimé à la souveraineté étatique, et il en va de même de celui du droit. Le statut équivoque des droits humains, auquel je faisais allusion plus haut, a pour pendant le statut également équivoque de la lex mercatoria moderne, dont les modes de formation défient toutes les conceptions dogmatiques concernant les sources du droit.

Il me semble par conséquent insuffisant, pour traiter de l'unité du monde, que l'on s'en tienne aux rapports de la politique et du droit. Je comprends bien l'intérêt que présente la thèse de l'autonomie (relative) du droit par rapport à un soulignement excessif de son moment politique, qui peut tourner à l'anti-juridisme. Mais faut-il, pour éviter le piège d'une interprétation trop exclusivement politique du droit, chercher chez les théoriciens du droit, et chez eux seulement, les moyens de comprendre sa fonction sociale? Ne risque-t-on pas d'ignorer, ce faisant, les imbrications complexes du politique, du droit et de l'économie ? Pour prendre la mesure de ce qu'il advient de la politique et du droit dans les conditions de la mondialisation contemporaine, l'opposition entre les situations de crise (de rupture) et la «situation normale » est manifestement trop simple. À ce niveau d'appréhension, le droit, précisément parce qu'il contribue de façon décisive à la structuration de l'économie, est un espace de dissensus. Du droit pluriel (par ses sources, par les instances qui le promulguent ou en gèrent l'application) du monde mondialisé, on ne peut dire qu'il est celui d'une société pacifiée. Ce qui me ramène à ma question initiale, relative aux droits humains. Dans un petit ouvrage, récemment paru, consacré à la lutte pour les droits sociaux globaux $^{1}$, les auteurs remarquent que le droit contemporain a un statut

1. Andreas Fischer-Lescano \& Kolja Möller: Der Kampf um globale soziale Rechte, Verlag Klaus Wagenbach, Berlin, $20 \mathrm{I} 2$. 
paradoxal, écartelé entre domination et émancipation, entre son instrumentalisation par les tenants d'une logique économique dont les effets sociaux sont souvent dévastateurs (on pense au rôle des gros cabinets d'avocats dans la défense juridique des activités prédatrices de grosses compagnies transnationales) et son usage possible par des populations dominées. Une grande partie des lignes de partage déterminantes pour le monde qui est le nôtre traverse aujourd'hui le droit, non pas seulement parce que le droit en général a pour fonction de résoudre les conflits, mais avant tout parce que le droit ne peut être un moyen d'émancipation qu'à la condition que sa définition soit constamment remise en cause. Ce qui veut dire: du point de vue des luttes d'émancipation, à l'échelle nationale, mais plus encore, aujourd'hui, à l'échelle mondiale, la "normalité » d'un ordre juridique établi est toujours illusoire. 